\title{
Snake-like Chromatin Cell Protein as an Indicator of Chronic Eye Rubbing in Patients with Keratoconus and Allergic Conjunctivitis
}

${ }^{1}$ Maria A Henriquez, ${ }^{2}$ Nadia Canorio, ${ }^{3}$ Carmen Maldonado, ${ }^{4}$ Mirel Rincon, ${ }^{5}$ Luis Izquierdo Jr

\begin{abstract}
Purpose: To evaluate the presence of snake-like chromatin (SLC) cell protein (related to eye rubbing) in patients with keratoconus or allergic conjunctivitis and in controls.

Materials and methods: Prospective experimental study included 193 eyes of 193 patients between January 2015 and July 2016; 86 had a diagnosis of new keratoconus, 88 were controls, and 19 had a diagnosis of allergic conjunctivitis and underwent impression cytology (IC). Specimens were stained with a combination of periodic acid-Schiff (PAS) and Gill's modified papanicolaou stains to detect the presence of SLC cell protein and morphological characterization of dry eye and allergic conjunctivitis. A questionnaire regarding eye-rubbing habits was administered.
\end{abstract}

Results: Of the patients, $56.97 \%$ (49/86 eyes) in the keratoconus group, $84.21 \%$ (16/19 eyes) in the allergy group, and $10.11 \%$ (9/89 eyes) in the control group reported rubbing their eyes. The SLC was detected in $24.41 \%$ (21/86), $73.68 \%$ (14/19), and $3.37 \%(3 / 89)$ of the keratoconus, allergy, and control groups respectively, who reported rubbing their eyes. In contrast, SLC was detected in $2.32 \%(2 / 86), 0 \%(0 / 19)$, and $1.12 \%(1 / 89)$ of the keratoconus, allergy, and control groups respectively, who reported not rubbing their eyes.

Conclusion: The presence of SLC cells was higher in keratoconus, allergy, and control group patients who reported rubbing their eyes. Keratoconus is independent of eye-rubbing habits and the presence of SLC cells.

Keywords: Conjunctivitis, Eye, Habit, Keratoconus, Rubbing.

How to cite this article: Henriquez MA, Canorio N, Maldonado C, Rincon M, Izquierdo L Jr. Snake-like Chromatin Cell Protein as an Indicator of Chronic Eye Rubbing in Patients with Keratoconus and Allergic Conjunctivitis. Int J Kerat Ect Cor Dis 2018;7(2):91-95.

Source of support: Nil

Conflict of interest: None

\section{INTRODUCTION}

Keratoconus is a corneal ectatic disorder characterized by bilateral conical protrusion and thinning. ${ }^{1}$ According

1,2,5 Associate Professor, ${ }^{3}$ Study Coordinator, ${ }^{4}$ Resident,

${ }^{1-5}$ Department of Research, Oftalmosalud Instituto de Ojos, Lima, Peru

Corresponding Author: Maria A Henriquez, Associate Professor, Department of Research, Oftalmosalud Instituto de Ojos, Lima, Peru, e-mail: mariale_1610@hotmail.com to the United States Eye bank and International Eye banking statistics, keratoconus represents the second most common reason for corneal transplantation. ${ }^{2}$ Its prevalence has been estimated with a fairly wide range, between $0.0003 \%$ and $2.34 \%$, and it is attributed to different geographical areas, genetics, nutrition habits, and ethnicities. $^{3}$

Despite the recent and great advances that have been made in the diagnosis and treatment of keratoconus, the exact cause of this disease is not known; however, it is attributed to being a multifactorial disease for which genetic, biomechanical, and environmental components play important roles. ${ }^{4}$ Significant risk factors have been described, including age (younger), gender (male), eye rubbing, atopia, parents education, positive family history, Down syndrome, Leber congenital amaurosis, and others. ${ }^{5-7}$ However, eye rubbing has been the focus in past years, and it has been questioned whether this is a risk factor or if it could be the cause of keratoconus. ${ }^{8-15}$

The snake cell protein is a nuclear morphologic alteration of the conjunctival epithelium. It is a condensation and segmentation of the chromatin. Because the morphological features look like a snake, it is called SLC. The SLC has been considered an indicator of mechanical stress on the ocular surface because a mechanical stimulus is able to alter the nuclear skeleton in this way. ${ }^{16,17}$

The aim of this study was to evaluate the presence of SLC cell protein related to chronic mechanical trauma induced by eye rubbing in patients with keratoconus or allergic conjunctivitis and in controls.

\section{MATERIALS AND METHODS}

This prospective experimental study included 193 new patients with keratoconus, 88 controls, and 19 with allergic conjunctivitis who attended the Oftalmosalud Instituto de Ojos (Lima, Peru), from January 2015 to July 2016. The study complied with the Declaration of Helsinki. The ethics committee and IRB of Oftalmosalud approved the study. Written informed consent was obtained from all patients.

Inclusion criteria for the keratoconus group were: New keratoconus diagnosis; not being a contact lens 
user during the past 8 months; diagnosis of keratoconus defined by the presence of one or more clinical signs (corneal stromal thinning, Vogt striae, Fleischer ring, scissoring of the red reflex, or oil droplet sign); and topographic/tomographic signs (an increased area of corneal power surrounded by concentric areas of decreasing power, inferior-superior power asymmetry, and skewing of the steepest radial axes above and below the horizontal meridian). ${ }^{18}$

Inclusion criteria for allergic conjunctivitis were at least one of the subjective, objective, and cytology inclusion criteria. Patients with seasonal allergic conjunctivitis, for which symptoms appear in a seasonal manner, and perennial allergic conjunctivitis, for which symptoms persist throughout the year, were included. The subjective symptom was itching. Objective symptoms included conjunctival hyperemia, dilated conjunctival vessels, and a conjunctival follicle under the lower palpebral conjunctival epithelium. For inclusion, the patients were required to be positive for eosinophil according to the IC of the conjunctiva. Only one eye for each patient was included in the study. ${ }^{19-21}$

Inclusion criteria for the control group were patients who attended the clinic for annual examinations; no ocular symptoms or ocular pathology; no irregular corneal patterns; no previous ocular surgery; no significant refractive errors; not being a contact lens user during the past 8 months; and no clinical and tomographic signs of keratoconus or scissoring on retinoscopy.

\section{IC Procedure}

During IC, a light microscope was used at $40 \times$ magnification. Under topical anesthesia, a strip of cellulose acetate filter paper was placed on the upper, lower, and tarsal bulbar conjunctiva for 5 seconds. The strips were fixed with 95\% ethanol, stained with PAS and hematoxylin, and then fixed to a slide.

\section{Dry Eye Assessment with IC}

Under the light microscope, the nucleus-to-cytoplasm ratio, intercellular spaces, nucleus morphology, epithelial cell morphology, cytoplasmic staining, and number of goblet cells were evaluated. Nelson's 1983 scale was used for classification. ${ }^{21}$

\section{Snake Cell Protein Assessment}

We observed the conjunctival cells obtained by IC and evaluated the presence of snake-like condensations of the nuclear chromatin of conjunctival epithelial cells in $1 \times$ $1 \mathrm{~mm}$ field, and considered positive if there were more than two cells with this type of condensation.

\section{Statistical Analysis}

Statistical analyses were performed using $\mathrm{R}$ software version 3.4.1. Pairwise comparisons were made between each of the proportions of the study groups using pairwise comparisons using Fisher's exact test with correction for multiple testing by Holm. All comparisons were performed using $\alpha=0.05$.

\section{RESULTS}

One hundred ninety-three eyes of 193 patients were included: 86 eyes in the keratoconus group, 19 eyes in the allergic conjunctivitis group, and 88 eyes in the control group. Mean ages of the keratoconus, allergy, and control groups were 25.12 [standard deviation (SD): 5.79], 24.5 (SD: 6.48), and 27.58 (SD: 5.8) years, respectively with no statistically significant differences between them ( $p>0.05)$. Table 1 shows the eye-rubbing habits, presence of SLC cells, and prevalence of dry eye in each group. Figure 1 shows the SLC of a keratoconus patient with an eye-rubbing habit of six times per week.

In the keratoconus group, 56.97\% (49/86) of the patients reported rubbing their eyes. Of these eyes, SLC was detected in $24.41 \%(21 / 86)$ of the eyes; the frequency of eye rubbing for those with keratoconus and the presence of SLC was 4.47 (SD: 1.47) days per week; SLC was no detected in $32.55 \%(28 / 86)$ of the patients who referred to rubbing their eyes, the frequency of eye rubbing in those patients was 2.03 (SD: 1.45) days/ week. Moreover, $43.02 \%$ (37/86) of keratoconus patients did not have an eye-rubbing habit; SLC was detected in two of these eyes.

In the allergy group, $84.21 \%(16 / 19)$ of patients reported rubbing their eyes; of these eyes, SLC was detected in $73.7 \%(14 / 19)$; the eye-rubbing frequency for those with an allergy and the presence of SLC was 4.42 (SD: 1.39) days per week, and for those without SLC was 2 (SD: 1.41) days/week. Furthermore, 15.78\% (3/19) of

Table 1: Eye-rubbing habits, positive presence of SLC cells and dry eye in each group

\begin{tabular}{lllllll}
\hline & Keratoconus & Allergy & Control & $p$ value & $p$ value & $p$ value \\
\hline $\mathrm{n}$ & 86 & 19 & 89 & & & \\
Positive habit for rubbing eye & $56.97 \%(49 / 86)$ & $84.21 \%(16 / 19)$ & $10.11 \%(9 / 89)$ & 0.04 & $<0.001$ & $<0.001$ \\
Positive presence of SLC & $26.74 \%(23 / 86)$ & $73.68 \%(14 / 19)$ & $4.49 \%(4 / 89)$ & $<0.001$ & $<0.001$ & $<0.001$ \\
Positive dry eye IC & $79.1 \%(68 / 86)$ & $78.9 \%(15 / 19)$ & $24.7 \%(22 / 89)$ & $>0.05$ & $<0.001$ & $<0.001$ \\
\hline
\end{tabular}

${ }^{*} p$ value for keratoconus and allergy; ${ }^{* *} p$ value for keratoconus and control; ${ }^{* *} p$ value for control and allergy 

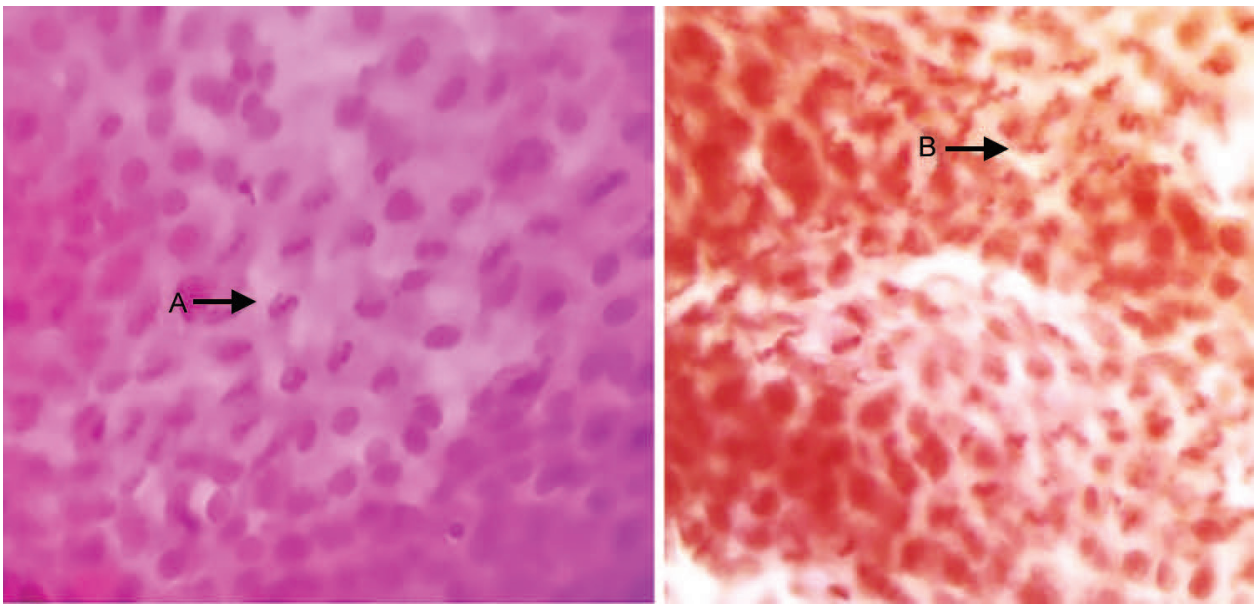

Fig. 1: SLC cell protein of a keratoconus patient with an eye-rubbing habit of 6 times per week

allergy patients did not have a habit of rubbing their eyes; SLC was detected in $0 \%(0 / 19)$ of these eyes.

In the control group, $10.11 \%(9 / 89)$ of the patients reported rubbing their eyes; of these eyes, SLC was detected in 3.37\% (3/89); the eye-rubbing frequency for those in the control group who rub their eyes and with SLC was 4 days per week (SD: 0.81); 7.86\% (7/89) without SLC and eye-rubbing habit had a frequency of eye rubbing of 1.2 days per week (SD: 0.44). Furthermore, $89.88 \%$ (80/89) of control group patients did not rub their eyes; SLC was detected in $1.12 \%(1 / 89)$ of these eyes.

\section{DISCUSSION}

Although in the past two decades, many advances have been made regarding keratoconus diagnosis and treatment, its cause is still unknown. ${ }^{5}$ Therefore, it has been postulated as a multifactorial disease. ${ }^{4,5,7,22,23} \mathrm{Mul}$ tiple studies have revealed an association with certain genes; ${ }^{22,24}$ however, it currently cannot be diagnosed based on genetic screening. ${ }^{23,24}$

Eye rubbing has been postulated as a significant risk factor by several authors. ${ }^{3,5,7,25-27}$ However, few studies ${ }^{8-15}$ have referred to eye rubbing as the cause of keratoconus; therefore, it has recently attracted attention. Mashor et al. ${ }^{9}$ reported three patients with Tourette syndrome and asymmetrical or unilateral keratoconus. Koenig et al. ${ }^{11}$ described bilateral recurrent keratoconus in a patient with self-induced keratoconus secondary to compulsive eye rubbing. Yeniad et al. ${ }^{12}$ reported a case of recurrent keratoconus after penetrating keratoplasty due to allergic conjunctivitis. Diniz et al. ${ }^{13}$ reported a case of unilateral keratoconus associated with continual eye rubbing due to nasolacrimal obstruction. Ioannidis et al. ${ }^{14}$ reported a case of unilateral keratoconus in a healthy child with chronic and persistent eye rubbing. Jafri et al. ${ }^{15}$ reported a series of cases of asymmetric keratoconus attributed to eye rubbing. Gatinel ${ }^{8}$ recently reported the hypothesis that keratoconus may never occur in a cornea that is not subjected to repeated mechanical trauma. Although most of these cases indicate eye rubbing as a cause of keratoconus, the majority of researchers did not perform genetic or inflammatory screenings for these patients. In our opinion, this is difficult to understand when there are studies ${ }^{27,28}$ that have reported a very low prevalence of keratoconus in a large series of vernal keratoconjunctivitis patients and a nonsignificant association between eye rubbing and keratoconus prevalence.

Eye-rubbing habits revealed that patients with allergic conjunctivitis had the highest tendency for eye rubbing (84.21\%) compared with those with keratoconus (56.97\%) and controls (10.11\%). In accordance with these results, the presence of SLC was significantly more frequent in the eyes of allergic conjunctivitis patients than in those with keratoconus and controls.

The results of the present study demonstrate that it is possible to determine whether patients are chronically rubbing their eyes by looking at the chromatin alteration in the conjunctival epithelial cells due to the mechanical effects of eye rubbing, thus indicating that keratoconus can occur in patients without eye rubbing and refuting the hypothesis of eye rubbing as an essential characteristic of keratoconus. It should be emphasized that our study does not demonstrate the relationship between eye rubbing and keratoconus progression, and it does not deny the effects of eye rubbing on the cornea. It is well known that eye rubbing induces changes in epithelial thickness, tear film, and keratometry. ${ }^{29,30}$ However, until now, it has not been possible to prove that keratoconic topographic alterations are due to the effects of eye rubbing or to intrinsic disease.

In this series, $43.02 \%$ of keratoconus patients reported not rubbing their eyes, and SLC was found in two of these eyes $(2.32 \%)$. In contrast, of the $57 \%$ of keratoconus patients who reported rubbing their eyes, SLC was present in 21 
(24.41\%) of them. Although the presence of SLC was greater in the eyes of patients with an eye-rubbing habit, there were 28 patients who reported eye rubbing but did not have SLC. This could be due to the presence of SLC is associated with chronic mechanical trauma. Patients with an eye-rubbing habit and SLC had an eye-rubbing frequency of 4.47 days per week, whereas keratoconus patients with an eye-rubbing habit but without SLC had an eye-rubbing frequency of 2.03 days per week. Although it is not possible to objectively measure how much the individuals are rubbing their eyes, our results suggest that the greater the mechanical trauma, the greater the probability of finding this nuclear alteration. However, two keratoconus eyes and one normal eye showed the presence of SLC in those without an eye-rubbing habit. This is in agreement with the work of Knop and Reale, ${ }^{17}$ who reported that SLC can be observed in a small proportion of the "normal" population, but the frequency is never as high as that for those experiencing chronic mechanical friction. The SLC could represent a way to objectively measure the impact of eye rubbing on the ocular surface. To the best of our knowledge, this is the first study that evaluates the presence of SLC cell protein related to chronic mechanical trauma induced by eye rubbing in patients with keratoconus.

The SLC is a nuclear alteration that occurs under pathological conditions and in different tissues. ${ }^{16,31,32}$ Although it is not a specific alteration that occurs only with eye rubbing, the presence of SLC accompanies mechanical irritation and is considered an indicator of mechanical stress on the ocular surface. ${ }^{17}$ Some studies have found it in the conjunctiva of contact lens users, thus arguing that the eyelid stroke generates repeated displacement of the contact lens with consequent friction against the bulbar conjunctival surface. ${ }^{17}$ In chronic dry eye patients, SLC is attributed to the increase of mechanical friction between the eyelid and the ocular surface. ${ }^{22,23}$ However, this has also been found with other conditions, such as eye surgery and is usually associated with chronic mechanical irritation or trauma. ${ }^{17}$

Some hypotheses regarding the cause of keratoconus related to eye rubbing are the release of inflammatory mediators, ${ }^{26}$ increased corneal temperature (thereby explaining corneal deformation), ${ }^{29}$ epithelial thinning, large intraocular pressure spikes, and others. ${ }^{33}$ However, it is difficult to prove that the mechanical stress induced by severe eye rubbing can cause structural changes to the cornea but not to the chromatin of conjunctival epithelial cells, which is altered by eye rubbing, contact lens use, and dry eye friction. .7,22,24

A limitation of our study was that SLC was evaluated based on epithelial conjunctival cells instead of corneal epithelial cells because SLC has been studied just in conjunctival cells. However, it is widely known that keratoconus eyes present not only alterations limited to the cornea but also tears, and that the conjunctiva expresses inflammatory alterations. Therefore, we believe that conjunctival epithelial cells are a reliable source for measuring the mechanical trauma induced by rubbing. In fact, patients are rubbing the eye, and not specifically the cornea.

With regard to dry eye, we show that patients with keratoconus have a much greater prevalence of dry eye than controls; however, the prevalence is very similar to that of those with allergic conjunctivitis. This supports the results of several studies that have shown a relationship between inflammation and keratoconus. ${ }^{26,34}$

In conclusion, this study shows that SLC cells have a higher frequency in patients with chronic eyerubbing habits. The SLC could be an objective indicator of mechanical trauma in epithelial conjunctival cells due to chronic eye rubbing. However, keratoconus can occur in a cornea that is not subjected to eye rubbing. Further studies are needed to demonstrate the association between eye rubbing and keratoconus progression.

\section{REFERENCES}

1. Rabinowitz YS. Keratoconus. Surv Ophthalmol 1998;42(4):297319.

2. Wong KH, Kam KW, Chen LJ, et al. Corneal blindness and current major treatment concern-graft scarcity. Int J Ophthalmol 2017;10(7):1154-1162.

3. Gordon-Shaag A, Millodot M, Shneor E. The epidemiology and etiology of keratoconus. Int J Keratoco Ectatic Corneal Dis 2012;1(1):7-15.

4. Gomes JA, Rapuano CJ, Belin MW, et al; Group of Panelists for the Global Delphi Panel of Keratoconus and Ectatic Diseases. Global consensus on keratoconus diagnosis. Cornea 2015;34(12):e38-e39.

5. Gordon-Shaag A, Millodot M, Kaiserman I, et al. Risk factors for keratoconus in Israel: a case-control study. Ophthalmic Physiol Opt 2015;35(6):673-681.

6. Naderan M, Shoar S, Rezagholizadeh F, et al. Characteristics and associations of keratoconus patients. Cont Lens Anterior Eye 2015;38(3):199-205.

7. Kok YO, Tan GF, Loon SC. Review: keratoconus in Asia. Cornea 2012;31(5):581-593.

8. Gatinel D. Eye rubbing, a sine qua non for keratoconus? Int J Kerat Ect Dis 2016;5(1):6-12.

9. Mashor RS, Kumar NL, Ritenour RJ, et al. Keratoconus caused by eye rubbing in patients with Tourette syndrome. Can J Ophthalmol 2011;46(1):83-86.

10. Balasubramanian SA, Pye DC, Willcox MD. Are proteinases the reason for keratoconus? Curr Eye Res 2010;35(3):185-191.

11. Koenig SB. Bilateral recurrent self-induced keratoconus. Eye Contact Lens 2008;34(6):343-344.

12. Yeniad B, Alparslan N, Akarcay K. Eye rubbing as an apparent cause of recurrent keratoconus. Cornea 2009;28(4): 477-479.

13. Diniz CM, Tzelikis PF, Rodrigues Júnior A, et al. Unilateral keratoconus associated with continual eye rubbing due to 


\section{IJKECD}

nasolacrimal obstruction-case report. Arq Bras Oftalmol 2005;68(1):122-125.

14. Ioannidis AS, Speedwell L, Nischal KK. Unilateral keratoconus in a child with chronic and persistent eye rubbing. Am J Ophthalmol 2005;139(2):356-357.

15. Jafri B, Lichter $H$, Stulting RD. Asymmetric keratoconus attributed to eye rubbing. Cornea 2004;23(6):560-564.

16. Jirsova K, Juklova K, Vesela V, et al. Morphological and immunocytochemical characterization of snake-like chromatin cells. Histol Histopathol 2006;21(4):355-360.

17. Knop E, Reale E. Fine structure and significance of snakelike chromatin in conjunctival epithelial cells. Invest Ophthalmol Vis Sci 1994;35(2):711-719.

18. Ambrósio R Jr, Caiado AL, Guerra FP, et al. Novel pachymetric parameters based on corneal tomography for diagnosing keratoconus. J Refract Surg 2011;27(10):753-758.

19. Takamura E, Uchio E, Ebihara N, et al. Japanese guidelines for allergic conjunctival diseases 2017. Allergol Int 2017;66(2): 220-229.

20. Leonardi A, Bogacka E, Fauquert JL, et al. Ocular allergy: recognizing and diagnosing hypersensitivity disorders of the ocular surface. Allergy 2012;67(11):1327-1337.

21. Gadkari SS, Adrianwala SD, Prayag AS, et al. Conjunctival impression cytology - a study of normal conjunctiva. J Postgrad Med 1992;38(1):21-23, 22A-22B.

22. Karolak JA, Gambin T, Pitarque JA, et al. Variants in SKP1, PROB1, and IL17B genes at keratoconus 5q31.1-q35.3 susceptibility locus identified by whole-exome sequencing. Eur J Hum Genet 2016;25(1):73-78.

23. Burdon KP, Vincent AL. Insights into keratoconus from a genetic perspective. Clin Exp Optom 2013;96(2):146-154.
24. Lauwen S, de Jong EK, Lefeber DJ, et al. Omics biomarkers in ophthalmology. Invest Ophthalmol Vis Sci 2017;58(6):BIO88BIO98.

25. Bawazeer AM, Hodge WG, Lorimer B. Atopy and keratoconus: a multivariate analysis. Br J Ophthalmol 2000; 84(8):834-836

26. Sharma N, Rao K, Maharana PK, et al. Ocular allergy and keratoconus. Indian J Ophthalmol 2013;61(8):407-409.

27. Caputo R, Versaci F, Pucci N, et al. Very low prevalence of keratoconus in a large series of vernal keratoconjunctivitis patients. Am J Ophthalmol 2016;172:64-71.

28. Millodot M, Shneor E, Albou S, et al. Prevalence and associated factors of keratoconus in Jerusalem: a cross-sectional study. Ophthalmic Epidemiol 2011;18(2):91-97.

29. McMonnies CW, Alharbi A, Boneham GC. Epithelial responses to rubbing-related mechanical forces. Cornea 2010;29(11):1223-1231.

30. Chervenkoff JV, Hawkes E, Ortiz G, et al. A randomized, fellow eye, comparison of keratometry, aberrometry, tear film, axial length and the anterior chamber depth after eye rubbing in non-keratoconic eyes. Eye Vis (Lond) 2017;4:19.

31. Marner K. 'Snake-like' appearance of nuclear chromatin in conjunctival epithelial cells from patients with keratoconjunctivitis sicca. Acta Ophthalmol (Copenh) 1980;58(5): 849-853.

32. Pienaar JG, Price HM. Ultrastructure and origin of the Anitschkow cell. Am J Pathol 1967;51(6):1063-1091.

33. McMonnies CW. Mechanisms of rubbing-related corneal trauma in keratoconus. Cornea 2009;28(6):607-615.

34. Galvis V, Sherwin T, Tello A, et al. Keratoconus: an inflammatory disorder? Eye (Lond) 2015;29(7):843-859. 